\title{
ChemComm
}

\section{Improved green-light-emitting pyrotechnic formulations based on tris(2,2,2-trinitroethyl)- borate and boron carbide}

50,9581

Received 18th June 2014

Accepted 5th July 2014

DOI: $10.1039 / c 4 c c 04616 a$

www.rsc.org/chemcomm

\author{
Thomas M. Klapötke, ${ }^{\star a}$ Burkhard Krumm, ${ }^{a}$ Magdalena Rusan ${ }^{a}$ and \\ Jesse J. Sabatini ${ }^{\star b}$
}

\begin{abstract}
Green-light-emitting pyrotechnic compositions based on tris(2,2,2trinitroethyl)borate (TNEB) and boron carbide have been investigated. The best performing formulations were found to be insensitive to various ignition stimuli, and exhibited very high spectral purities and luminosities compared to previously reported green-light-emitting formulations.
\end{abstract}

Traditional green-light-emitting pyrotechnic formulations rely on a combination of barium compounds and chlorine donors to achieve a suitable green-light-emitting species. ${ }^{1}$ In these mixtures, barium nitrate is combined with a chlorine donor such as poly(vinyl) chloride to form metastable barium(I) chloride as the green-light-emitting species. ${ }^{1}$ Spectral purities of barium-based green-light-emitting illuminants are typically in the low-to-high $60 \%$ range. This relatively low spectral purity is a result of large amounts of white-light-emitting incandescent particles (i.e. $\mathrm{MgO}, \mathrm{BaO}$ ) formed during the combustion of a given formulation. Unfortunately, barium compounds are suspected cardiotoxins and have been linked to hazards associated with occupational health. ${ }^{2}$ Furthermore, there is concern that the combustion of chlorine donors such as PVC leads to the production of significant amounts of carcinogenic materials such as polychlorinated biphenyls (PCBs), polychlorinated dibenzodioxins (PCDDs), and polychlorinated dibenzofurans (PCDFs). ${ }^{3}$ Thus, the removal of barium- and chlorine-containing materials from green-light-emitting pyrotechnic formulations is of high interest in "greening" this class of green-light-emitting pyrotechnic formulations.

In 2011, Sabatini et al. developed a barium- and chlorinefree green-light-emitting pyrotechnic formulation by burning a mixture of potassium nitrate/boron carbide/epoxy binder. ${ }^{4}$

\footnotetext{
${ }^{a}$ Department of Chemistry, Energetic Materials Research, Ludwig-Maximilian

University of Munich, Butenandtstr. 5-13, D-81377 Munich, Germany.

E-mail: tmk@cup.uni-muenchen.de; Fax: +49892180 77492;

Tel: +4989218077491

${ }^{b}$ Pyrotechnics Technology and Prototyping Division US Army RDECOM-ARDEC, Picatinny Arsenal, NJ 08706-5000, USA.E-mail: jesse.j.sabatini.civ@mail.mil; Fax: $+1-973-724-7375$
}

Green-light-emission occurred due to the formation and emission of metastable boron dioxide $\left(\mathrm{BO}_{2}\right)$ as opposed to the traditional barium(I) chloride species. While this mixture gives decent greenlight-emission and is very insensitive to impact friction and electrostatic discharge, it has a relatively low spectral purity. Low spectral purities are a consequence of "washing out" of the flame colour. This phenomenon likely occurred due to the presence of high levels of potassium nitrate, which produces white-lightemitting $\mathrm{KOH}^{5}{ }^{5}$ Therefore, it was believed that replacement of potassium nitrate with a metal-free oxidizer would improve the colour properties through the minimization of incandescent particle emission. Although it was initially postulated that mixtures of ammonium nitrate/boron carbide or ammonium dinitramide/boron carbide may result in high quality green-lightemission, ${ }^{5,6}$ attempts to produce green light by these means were unsuccessful. Since the production of metastable $\mathrm{BO}_{2}$ favours green-light-emission, attention was then turned to a boron-containing oxidizer to maximize the quality of a green-lightemitting flame based on boron carbide. Tris(2,2,2-trinitroethyl)borate (TNEB), which was synthesized previously, ${ }^{7}$ was considered to be the compound of interest in this study. TNEB contains both a boron centre and trinitroethyl energetic ligands. With an oxygen balance of +13.1 , TNEB was determined to be a suitable oxidizing material for this research investigation.

The green-light-emitting formulation containing potassium nitrate and boron carbide reported by Sabatini et al. ${ }^{4}$ was reinvestigated with the herein used equipment to establish a relevant data point (Table 1). This green-light-emitting formulation had a spectral purity of $69 \%$ (Table 2). It should be noted that TNEB (Fig. 1) is a moisture sensitive material, owing to the highly reactive nature and the vacant p-orbital of the boron centre. When synthesized, the material decomposes after several

Table 1 Formulation 1

\begin{tabular}{llll}
\hline Formulation & $\mathrm{KNO}_{3}[\mathrm{wt} \%]$ & $\mathrm{B}_{4} \mathrm{C}[\mathrm{wt} \%]$ & $\begin{array}{l}\text { Epon 828/Epikure } \\
3140[\mathrm{wt} \%]\end{array}$ \\
\hline $\mathbf{1}$ & 83 & 10 & 7
\end{tabular}


Table 2 Color properties of formulation 1

\begin{tabular}{llllll}
\hline Formulation & $\mathrm{BT}^{a}[\mathrm{~s}]$ & $\mathrm{DW}^{b}[\mathrm{~nm}]$ & $\mathrm{SP}^{c}[\%]$ & $\mathrm{LI}^{d}[\mathrm{~cd}]$ & $\mathrm{LE}^{e}\left[\mathrm{~cd} \mathrm{~s} \mathrm{~g}^{-1}\right]$ \\
\hline $\mathbf{1}$ & 6 & 560 & 69 & 25 & 250
\end{tabular}

${ }^{a} \mathrm{BT}=$ Burn time. ${ }^{b} \mathrm{DW}=$ Dominant wavelength. ${ }^{c} \mathrm{SP}=$ Spectral purity. ${ }^{d} \mathrm{LI}=$ Luminous intensity. ${ }^{e} \mathrm{LE}=$ Luminous efficiency.

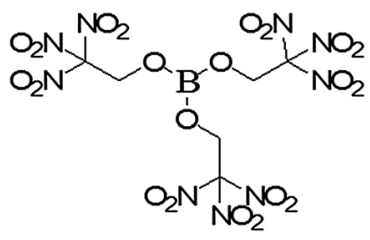

Fig. 1 Structure of tris(2,2,2-trinitroethyl)borate.

days of storage. When preparing pyrotechnic mixtures containing this oxidizer, it is recommended that non-basic materials be used to prevent any undesired side-reactions from occurring with TNEB. Therefore, the use of epoxy-based binder systems that typically employ polyamine-based curing agents is not recommended.

To help minimize the decomposition of TNEB, and to assist in providing sealant-like properties for the TNEB-containing formulations, paraffin was added to the formulations in lieu of the polyamine-based binder system. Mixing all solid ingredients in liquid paraffin offered a moisture resistant quality to the formulations detailed in Table 3 . The presence of magnesium in formulations $\mathbf{2}$ and $\mathbf{3}$ was critical in order to maintain a high combustion temperature. Green-light-emission was not observed when magnesium was omitted from these formulations.

The performances of both mixtures and their energetic and thermal properties are summarized in Table 4. Formulations 2 and 3 burned with little smoke, and yielded an intensive green flame (Fig. 2). These formulations reveal respective spectral purities of $86 \%$ and $85 \%$, which exceed the spectral purity of formulation 1 due to the absence of large quantities of potassium-based white-lightemission. The luminous intensities of formulations 2 and 3 are appreciably higher than the luminosity observed in formulation $\mathbf{1}$, a phenomenon due to the presence of magnesium in the former

Table 3 Formulations 2 and 3

\begin{tabular}{lllll}
\hline & TNEB $[\mathrm{wt} \%]$ & $\mathrm{B}_{4} \mathrm{C}[\mathrm{wt} \%]$ & $\mathrm{Mg}[\mathrm{wt} \%]$ & Paraffin $[\mathrm{wt} \%]$ \\
\hline $\mathbf{2}$ & 79 & 10 & 4 & 7 \\
3 & 75 & 10 & 8 & 7
\end{tabular}

Table 4 Color performances and energetic and thermal properties of formulations 2 and 3

\begin{tabular}{lllllllll}
\hline & $\mathrm{BT}^{a}$ & $\mathrm{DW}^{b}$ & $\mathrm{SP}^{c}$ & $\mathrm{LI}^{d}$ & $\mathrm{LE}^{e}$ & $\mathrm{IS}^{f}$ & $\mathrm{FS}^{g}$ & $T_{\mathrm{dec}^{h}}$ \\
Formulation & {$[\mathrm{s}]$} & {$[\mathrm{nm}]$} & {$[\%]$} & {$[\mathrm{cd}]$} & {$\left[\mathrm{cd} \mathrm{s} \mathrm{g}^{-1}\right]$} & {$[\mathrm{J}]$} & {$[\mathrm{N}]$} & {$\left[{ }^{\circ} \mathrm{C}\right]$} \\
\hline 2 & 7 & 561 & 86 & 50 & 583 & $>40$ & $>360$ & 170 \\
3 & 4 & 562 & 85 & 89 & 593 & $>40$ & $>360$ & 168
\end{tabular}

${ }^{a} \mathrm{BT}=$ Burn time. ${ }^{b} \mathrm{DW}=$ Dominant wavelength. ${ }^{c} \mathrm{SP}=$ Spectral purity. ${ }^{d} \mathrm{LI}=$ Luminous intensity. ${ }^{e} \mathrm{LE}=$ Luminous efficiency. ${ }^{f} \mathrm{IS}=$ Impact sensitivity.

${ }^{g}$ FS = Friction sensitivity. ${ }^{h} T_{\text {dec }}=$ Temperature of decomposition.

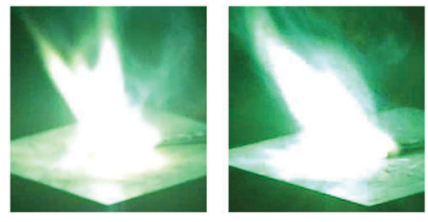

Fig. 2 Formulation 2 (left) and formulation $\mathbf{3}$ (right) at mid-burn.

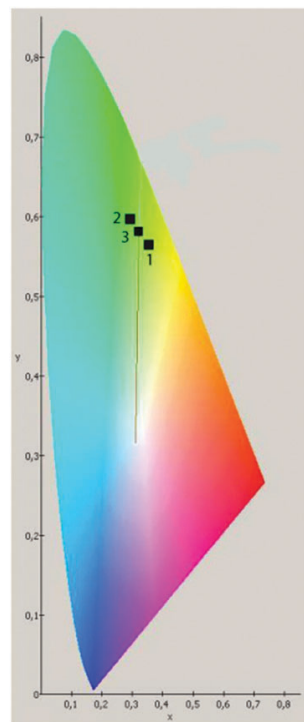

Fig. 3 CIE 1931 chromaticity diagram of formulations 1-3.

formulations. Formulation 2 was observed to be the best of the TNEB-based formulations tested on the basis of the performance obtained. Formulation 2 not only had an equivalent burn time to formulation $\mathbf{1}$, but it also surpassed the latter formulation in all performance categories by wide margins. Further pictorial evidence of the superior spectral purities of formulations $\mathbf{2}$ and 3 is provided in the CIE 1931 chromaticity diagram (Fig. 3).

Formulations 2 and 3 were found to respond to various ignition stimuli, each having an impact sensitivity higher than $40 \mathrm{~J}$ and a friction sensitivity in excess of $360 \mathrm{~N}$. The respective decomposition temperatures were $170{ }^{\circ} \mathrm{C}$ and $168{ }^{\circ} \mathrm{C}$.

In summary, a green-light-emitting pyrotechnic formulation with high performance and spectral purity has been obtained by means of replacing potassium nitrate with TNEB in the presence of boron carbide, magnesium, and paraffin wax. In particular, formulation 2 exceeds the performance of potassium nitrate-based formulation $\mathbf{1}$ in all categories, while formulation $\mathbf{3}$ yields the highest overall luminosity. The aforementioned compositions are very insensitive to impact and friction. Although further study is needed to address potential concerns associated with moisture sensitivity, the research is of potential interest to those in the pyrotechnics community concerned with finding environmentally friendly alternatives to barium- and chlorine-based green-lightemitting pyrotechnics of high luminosity and spectral purity.

Caution! Tris(2,2,2-trinitroethyl) borate is an energetic material and formulations 1-3 are energetic formulations with high sensitivity towards heat, impact and friction. Although no 
incidents occurred during preparation and manipulation, additional proper protective precautions like face shield, leather coat, earthed equipment and shoes, Kevlar gloves, and ear plugs should be used when undertaking work with these compounds. ${ }^{8}$

TNEB was synthesized according to the literature procedure. ${ }^{7}$ Boron carbide, amorphous boron, potassium nitrate, and paraffin were purchased from Aldrich and were used as received. The pyrotechnical compositions were prepared by grinding all substances in a mortar. The mixture was then introduced slowly into warm liquid paraffin. After cooling to room temperature, the mixtures were grinded again. Pellets of $0.6 \mathrm{~g}$ each were pressed using a consolidation dead load of $2000 \mathrm{~kg}$. The pellets were dried overnight at ambient temperature. The controlled burn was filmed using a digital video camera recorder (SONY, DCRHC37E). The performance of each composition was evaluated with respect to color emission, smoke generation, and the amount of solid residues. Spectrometric measurements were performed using a HR2000+ES spectrometer equipped with an ILX511B linear silicon CCD-array detector and included software from Ocean Optics with a detector-sample distance of 1 meter. The dominant wavelength and spectral purity were measured based on the 1931 CIE method using illuminant $\mathrm{C}$ as the white reference point. Luminous intensities and luminous efficiencies were determined using pellets of $0.6 \mathrm{~g}$ each. Five samples were measured for each formulation and all given values are averaged based on the full burn of the mixture. Decomposition points were measured using a Linseis PT10 DSC at heating rates of $5{ }^{\circ} \mathrm{C} \mathrm{min}^{-1} \cdot{ }^{9}$ The impact $^{10}$ and friction ${ }^{11}$ sensitivity was determined using a BAM drop hammer and a BAM friction tester. The sensitivities of the compounds are indicated according to the UN Recommendations on the Transport of Dangerous Goods $(+):{ }^{12}$ impact: insensitive $>$ $40 \mathrm{~J}$, less sensitive $>35 \mathrm{~J}$, sensitive $>4 \mathrm{~J}$, very sensitive $<4 \mathrm{~J}$; friction: insensitive $>360 \mathrm{~N}$, less sensitive $=360 \mathrm{~N}$, sensitive $<$ $360 \mathrm{~N}>80 \mathrm{~N}$, very sensitive $<80 \mathrm{~N}$, extremely sensitive $<10 \mathrm{~N}$.

Financial support of this work by the Ludwig-Maximilian University of Munich (LMU), the U.S. Army Research Laboratory (ARL) under grant no. W911NF-09-2-0018, the Armament Research, Development and Engineering Center (ARDEC) under grant no. W911NF-12-1-0467, and the Office of Naval Research (ONR) under grant no. ONR.N00014-10-1-0535 and ONR.N00014-12-1-0538 is gratefully acknowledged. The authors acknowledge collaborations with Dr Mila Krupka (OZM Research, Czech Republic) in the development of new testing and evaluation methods for energetic materials and with Dr Muhamed Suceska (Brodarski Institute, Croatia) in the development of new computational codes to predict the detonation and propulsion parameters of novel explosives. We are indebted to and thank Drs Betsy M. Rice and Brad Forch (ARL, Aberdeen, Proving Ground, MD) for many inspired discussions. The Cusanuswerk is gratefully acknowledged for the award of a $\mathrm{PhD}$ scholarship (M. Rusan).

\section{Notes and references}

1 (a) G. Steinhauser and T. M. Klapötke, Angew. Chem., Int. Ed., 2008, 47, 3330; (b) J. A. Conkling, Chemistry of Pyrotechnics: Basic Principles and Theory, Taylor \& Francis Group, New York, 1985, 156; (c) G. Steinhauser and T. M. Klapötke, J. Chem. Educ., 2010, 87, 150. 2 (a) R. Hicks, L. Q. Caldas, P. R. Dare and P. J. Hewitt, Arch. Toxicol. Supplement, 9, 415-420; (b) A. L. Reeves, Handbook on the Toxicology of Metals, Elsevier/North Holland Biomedical Press, New York, 1979, pp. 321-328.

3 D. Dyke and P. Coleman, Organohalogen Compounds, 1995, 24, 213. 4 J. J. Sabatini, J. C. Poret and R. N. Broad, Angew. Chem., Int. Ed., 2011, 50, 4624.

5 (a) T. M. Klapötke, M. A. Rusan, J. Stierstorfer, Proceedings of the $38^{\text {th }}$ International Pyrotechnic Seminars, Denver, CO, 2012, 527-550; (b) J. J. Sabatini, in Green Energetic Materials, ed. T. Brinck, Wiley-VCH, Weinheim, 2014, 78.

6 T. M. Klapötke, M. A. Rusan, and J. Stierstorfer, Proceedings of the $38^{\text {th }}$ International Pyrotechnic Seminars, Denver, CO, 2012, 527-550.

7 T. M. Klapötke, B. Krumm and R. Moll, Chem. - Eur. J., 2013, 19, 12113.

8 T. M. Klapötke, B. Krumm, F. X. Steemann and G. Steinhauser, Safety Science, 2010, 48, 28-34.

9 http://www.linseis.com.

10 NATO standardization agreement (STANAG) on explosives, impact sensitivity tests, no. 4489, 1st edn, Sept. 17, 1999.

11 NATO standardization agreement (STANAG) on explosive, friction sensitivity tests, no. 4487, 1st edn, Aug. 22, 2002.

12 UN Recommendations on the Transport of Dangerous Goods, Model Regulations, 15th edn, United Nations, New York a. Geneva, 2007. 